\title{
Mild and Versatile Functionalization of Nacre-Mimetic Cellulose Nanofibrils/Clay Nanocomposites by Organocatalytic Surface Engineering
}

\author{
Rana Alimohammadzadeh, Lilian Medina, Luca Deiana, Lars A. Berglund, and Armando Córdova*
}

Cite This: ACS Omega 2020, 5, 19363-19370

Read Online

ACCESS | Llll Metrics \& More | 国 Article Recommendations | st Supporting Information
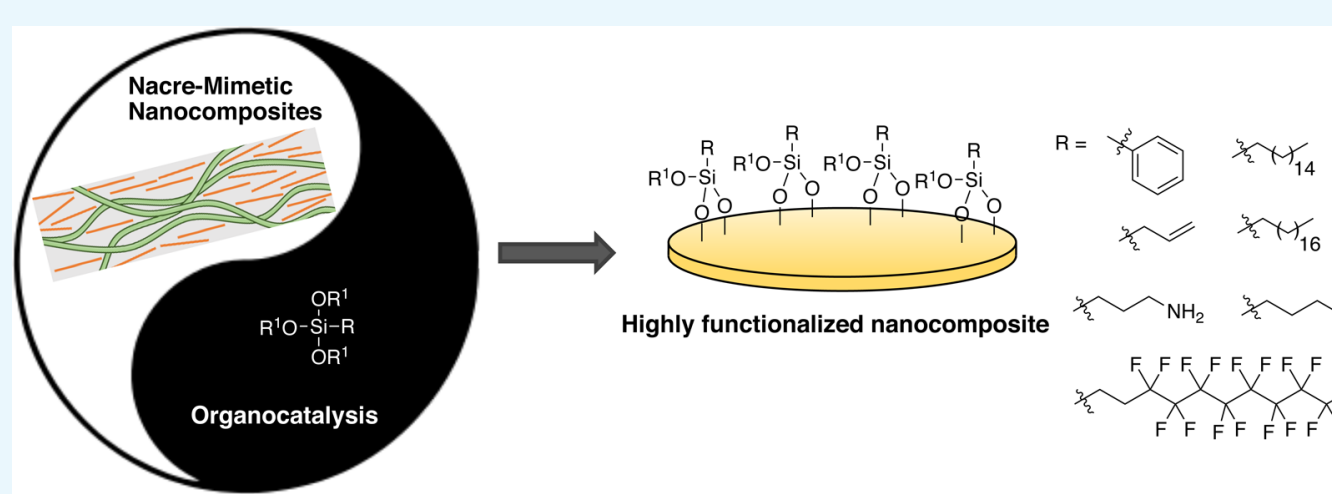

Highly functionalized nanocomposite

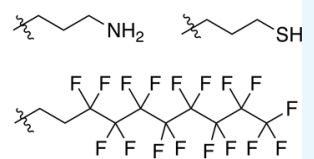

ABSTRACT: Development of surface-engineering strategies, which are facile, versatile, and mild, are highly desirable in tailor-made functionalization of high-performance bioinspired nanocomposites. We herein disclose for the first time a general organocatalytic strategy for the functionalization and hydrophobization of nacre-mimetic nanocomposites, which includes vide supra key aspects of surface engineering. The merging of metal-free catalysis and the design of nacre-mimetic nanocomposite materials were demonstrated by the organocatalytic surface engineering of cellulose nanofibrils/clay nanocomposites providing the corresponding bioinspired nanocomposites with good mechanical properties, hydrophobicity, and useful thia-, amino, and olefinic functionalities.

\section{INTRODUCTION}

Nature has the ability of producing lightweight materials with robust stability and excellent properties from simple building blocks. Thus, material scientists and engineers have gathered important lessons from nature on fabrication of lightweight materials with exceptional properties. ${ }^{1-3}$ In this context, nacre has gained significant research interest because it has exceptional stability and excellent properties derived from its unique brick-and-mortar structure, which comprises wellaligned inorganic platelets "glued" together by an organic matrix (chitin and proteins). ${ }^{4-6}$ Despite the many types of inorganic "bricks", which have been investigated when mimicking the nacre's brick-and-mortar structure, the present research is on clay platelets of nanometer thickness. ${ }^{7-11}$ Clay is also useful in reinforcing other types of composites. ${ }^{12,13}$ In seminal work, Kotov and co-workers introduced a general concept of nacre-mimetic materials for clay nanoplatelet-neat polymer systems. ${ }^{7}$ It is based on forming micron-thick films by sequential deposition of clay and polyelectrolytes. Another ground-breaking work is the introduction of cross-linking to reach excellent material properties with unprecedented levels of Young's modulus (>100 GPa). ${ }^{8}$ A novel core-shell approach was introduced by Walther and co-workers. ${ }^{13,14}$ Here, a polymer is absorbed on the clay nanoplatelets and subsequently the nonsorbing polymer is removed by centrifugation. This allows for the formation of core-shell particles with a minimum amount of polymer (ideally a monolayer) and the possibility of scalability. Most of the videsupra nacre-mimetic systems are built up of montmorillonite (MTM) as the bricks and a fossil-based polymer (e.g., poly(vinyl alcohol) (PVA) and poly(diallyldimethylammonium chloride) (PDDA)) as the mortar. An elegant and sustainable approach for constructing nacremimetic nanocomposite systems using cellulose nanofibrils (CNFs) as the renewable mortar instead of fossil-based polymers such as PVA and PDDA was introduced. ${ }^{15-22}$ The CNFs, which are the primary reinforcement component in the wood cell wall, have been derived from delignified wood pulp fibers and provide good mechanical properties to lightweight

Received: March 4, 2020

Accepted: June 12, 2020

Published: June 29, 2020 


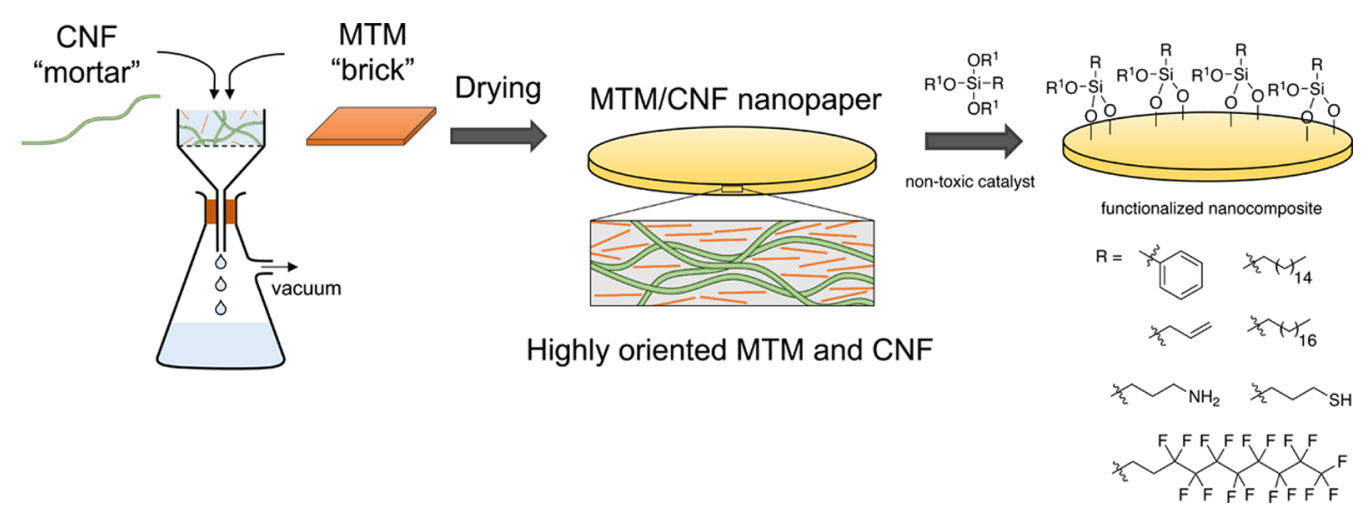

Figure 1. Schematic picture of the processing method for nanocomposite preparation combined with a catalytic modification approach.

composite materials. ${ }^{23-25} \mathrm{CNFs}$ also provide unique characteristics in the shape of the nanoscale geometry (diameter 3-15 $\mathrm{nm}$, length $0.7-2 \mu \mathrm{m}$ ) and beneficial mechanical properties of the individual fibrils (crystal modulus $\approx 137 \mathrm{GPa}$, tensile strength $\approx 2-6 \mathrm{GPa}$, low density $\left.\approx 1.6 \mathrm{~g} \mathrm{~cm}^{-1}\right){ }^{26,27}$ Moreover, modifications of the renewable CNFs can allow for surface functionalization of the nanofibril, providing CNFs with tailored characteristics (e.g., charge, size, and surface chemistry). ${ }^{28,29}$ However, the CNF surface is highly moisturesensitive as it is a natural polysaccharide with a multitude of hydroxyl groups. Moisture and water-sensitivity is also an issue for nacre-inspired clay-polymer nanocomposites in general, since they are mostly based on water soluble polymers such as PVA with an intrinsic hydrophilicity and high moisture sorption. Nacre-mimetics can also be made using graphene and ceramic particles as reinforcing agents, also exhibiting intrinsic water sensitivity. ${ }^{30,31}$ This is also the case when constructing nacre-like materials by, for example, 3Dprinting. ${ }^{32}$ In addition, the possibility for tailor-made surface properties is also highly desirable when looking for industrial and biomedical applications of clay nacre-mimetic composites. Today there are methods for making nacre-mimetic materials hydrophobic relying on, for example, covalent cross-linking or ionic supramolecular bonds. ${ }^{20,33-37}$ However, there is a lack of a general technology that in addition to hydrophobicity can install a large variety of functional groups. In addition, many disclosed methods are not practical and rather difficult to scale up for industrial applications. To address these challenges, we have developed an eco-friendly and general method for direct modification of nacre-mimetic nanocomposites. The MTM/ CNF system was chosen since it is sustainable and can serve as a general model for both renewable (e.g., MTM/polysaccharide) and fossil-based nacre-mimetic systems (e.g., MTM/ PVA).

"Organocatalysis" portrays the acceleration of chemical reactions through the addition of a substoichiometric quantity of an organic compound. The interest in this field has increased spectacularly during this century as a result of both the novelty of the concept and, more importantly, the fact that the efficiency and selectivity of many organocatalytic reactions can improve established organic reactions. In this context, we have developed scalable organocatalytic methods for direct esterification and silylation of polysaccharides. ${ }^{38-43}$ In fact, these metal-free transformations have been performed on a multi-ton scale by industry, which includes preparing waterrepellent textiles by organocatalytic silylations (e.g., OrganoTex). ${ }^{41}$ Here, the use of a metal-free catalyst significantly accelerates the rate of reaction. ${ }^{42,43}$ In addition, silanes are highly useful reagents in surface engineering of different inorganic materials (e.g., modification of metal surfaces for adhesives, silica nanoparticles, and glass fibers). The merging of organocatalysis with the design of nacre-mimetic materials should allow for a synergistic expansion of the design and application of this type of bioinspired future materials in a variety of industrial applications (e.g., coatings for buildings, spaceships, and biomedical implants). Herein, we disclose a highly versatile strategy for functionalization and hydrophobization of nacre-mimetic nanocomposites derived from simple building blocks using naturally occurring catalysts.

\section{RESULTS AND DISCUSSION}

Processing and Catalytic Modification. The fabricated MTM/CNF clay nanopaper composites are prepared by scalable vacuum-filtration and drying procedures similar to paper-making (Figure 1a). The enzymatically derived CNFs are on average $6.6 \mathrm{~nm}$ in diameter with a length of about $1 \mu \mathrm{m}$. The processing starts with the preparation of separate suspensions of CNF and MTM. In the latter case, only the highly stable exfoliated MTM supernatant fraction obtained after centrifugation is used. The mixed MTM and CNF suspension shows optical transparency; the filtration is relatively fast in the beginning but slows down as the MTM/ CNF nanocomposite film starts to form on top of the filtration membrane. Next, the resulting wet nanocomposite is dried to form a strong, transparent, and flexible film of MTM "bricks" dispersed in a CNF "mortar" inspired by nacre. ${ }^{44}$ With these results in hand, we explore the merging of nacre-mimetic nanocomposite fabrication with organocatalytic surface modification (Figure 1).

We began an initial catalyst screen for the hexadecyltrimethoxysilane (C16Si) and (3-thiapropyl)trimethoxysilane (TPSi) modification of MTM/CNF nanocomposite films. Natural organic acids were investigated as catalysts, since we have previously used them as Brønsted acid catalysts for direct surface modification of cellulose with various fatty acids, epoxides, polyesters, and silanes (Scheme 1). ${ }^{38-43}$

\section{Scheme 1. Brønsted Acid-Catalyzed Silylation}

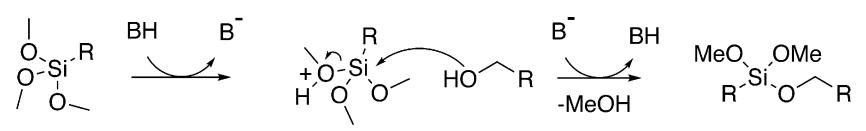

$\mathrm{BH}=$ Brønsted Acid Catalyst 
The organocatalytic reactions proceeded smoothly and the highest contact angles of the corresponding modified MTM/ CNF nanocomposites were obtained when using citric acid or L-tartaric acid as the catalysts (Table 1).

Table 1. Initial Screening for Surface-Modification of MTM/CNF ${ }^{a}$

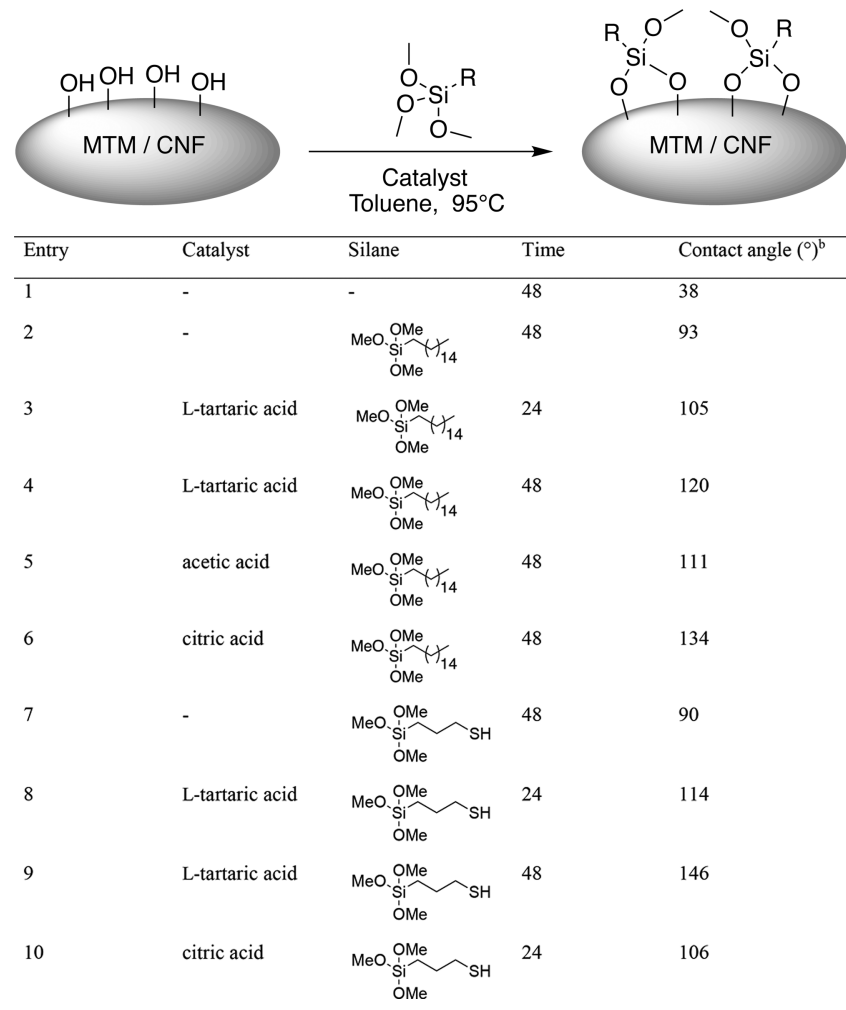

${ }^{a}$ The silane derivative ( 3 equiv, based on CNFs) and nanocomposites, which contains CNFs ( 1 equiv), were placed in an oven-dried reaction vessel containing the catalyst $(5 \mathrm{~mol} \%)$ and dry toluene $(50 \mathrm{~mL})$. After heating the reaction mixture for the time shown, the temperature was decreased to room temperature and toluene was decanted. Next, the modified nanocomposite was washed with acetone $(4 \times 50 \mathrm{~mL})$ and put in a desiccator and dried under reduced pressure. ${ }^{b}$ Contact angle error less than $0.5^{\circ}$.

Thus, significantly increasing the contact angle from the unmodified MTM/CNF film (entry 1, Figure 2a). For example, the contact angle is $134^{\circ}$ for the C16Si-MTM/
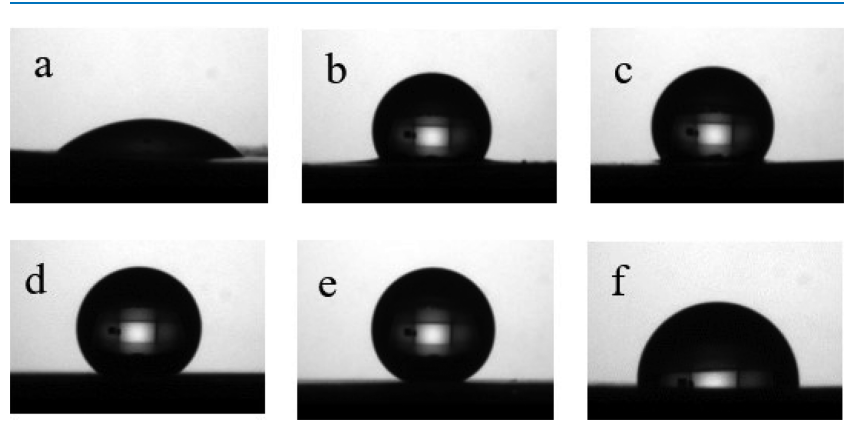

Figure 2. Water contact angle pictures of (a) MTM/CNF, (b) C16SiMTM/CNF, (c) AllylSi MTM/CNF, (d) TPSi- MTM/CNF, (e) $(1 \mathrm{H}, 1 \mathrm{H}, 2 \mathrm{H}, 2 \mathrm{H}$-heptadecaflurodecyl)Si-MTM/CNF, and (f) TPSiMTM/CNF modified without the catalyst.
CNF using citric acid as the catalyst (entry 6, Figure $2 \mathrm{~b}$ ) and the contact angle is $146^{\circ}$ for the TPSi-MTM/CNF using Ltartaric acid as the catalyst (entry 9, Figure $2 \mathrm{~d}$ ). In comparison, the reaction without catalyst is much slower and gave a contact angle of $90^{\circ}$ (entry 7, Figure $2 \mathrm{f}$ ).

In Table 2, the results from the simple and mild organocatalytic modification of MTM/CNF films with a

Table 2. Organocatalytic Surface Modification of MTM/ $\mathrm{CNF}^{a}$

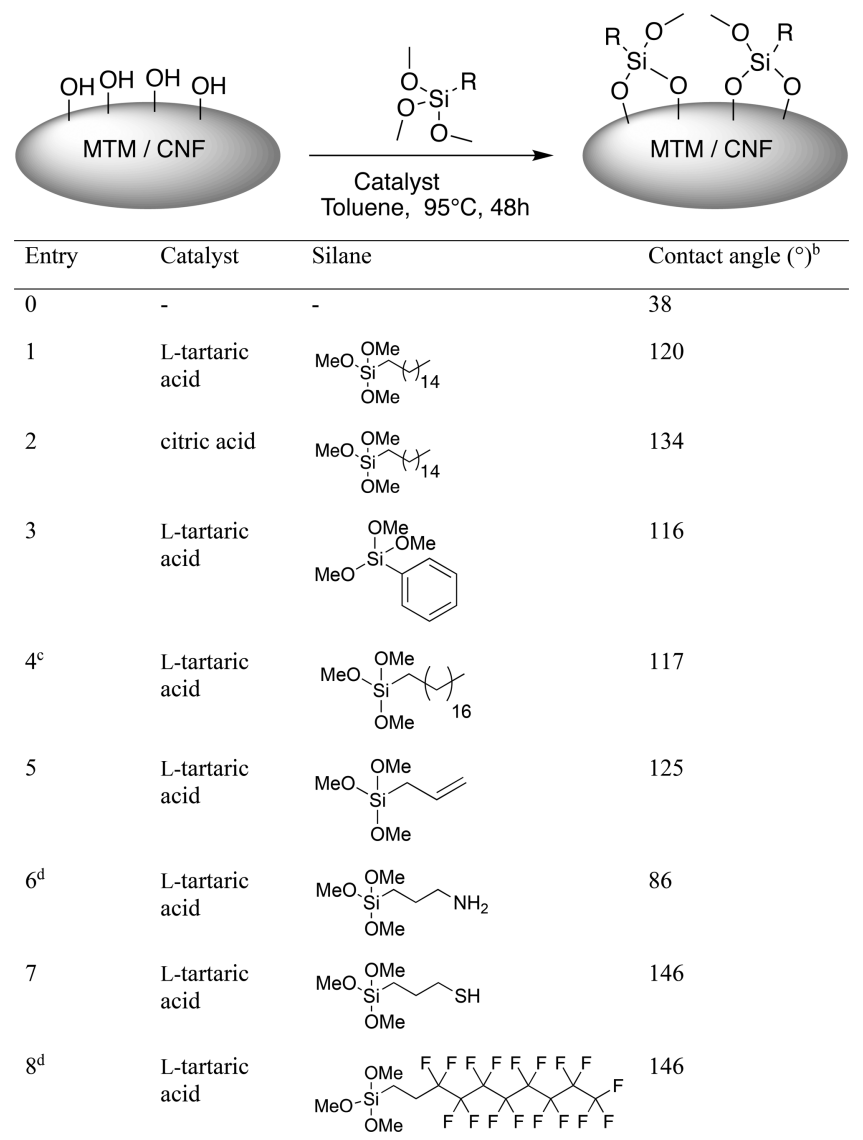

${ }^{a}$ The silane derivative (3 equiv, based on CNFs) and nanocomposites, which contains CNFs ( 1 equiv), were placed in an oven-dried reaction vessel containing the catalyst $(5 \mathrm{~mol} \%)$ and dry toluene $(50 \mathrm{~mL})$. After heating the reaction mixture for the time shown, the temperature was decreased to room temperature and toluene was decanted. Next, the modified nanocomposite was washed with acetone $(4 \times 50 \mathrm{~mL})$ and put in a desiccator and dried under reduced pressure. ${ }^{b}$ Contact angle error less than $0.5^{\circ} .{ }^{c}$ Reaction time is $24 \mathrm{~h} .{ }^{d}$ Reaction time is $6 \mathrm{~h}$.

variety of functional silanes using manly L-tartaric acid as the catalyst are shown. For example, when using organosilanes with $\mathrm{Ph}$, alkyl, allyl, and thiapropyl functionalities, the corresponding MTM/CNF-films became hydrophobic. The synthesized AllylSi MTM/CNF film had a contact angle of $125^{\circ}$ (entry 5, Figure 2c). The catalytic reaction with $(1 \mathrm{H}, 1 \mathrm{H}, 2 \mathrm{H}, 2 \mathrm{H}$-heptadecaflurodecyl $) \mathrm{Si}$ was much faster than the other silanes in reaching a high contact angle (entry 8 ). However, it is noteworthy that the TPSi-MTM/CNF (entry 7) had the same contact angle as a $(1 \mathrm{H}, 1 \mathrm{H}, 2 \mathrm{H}, 2 \mathrm{H}$ heptadecaflurodecyl)Si-modified MTM/CNF film (entry 8, Figure 2e). Thus, there is a chance of reaching similar levels of hydrophobicity without the use of an environmentally harmful 
perfluorocarbon functional group. ${ }^{45}$ It should be noted that the thia- and allyl-functional groups are very useful in "click chemistry" ${ }^{\$ 6,47}$ transformations and should allow for further degree of diversification, ${ }^{48,49}$ as well as selective attachment of polymers and UV-active groups to the nacre-mimetic nanocomposite films. The silane-modified MTM/CNF films exhibited the same type of flexibility and transparency after the catalytic surface modifications (Figure 3).

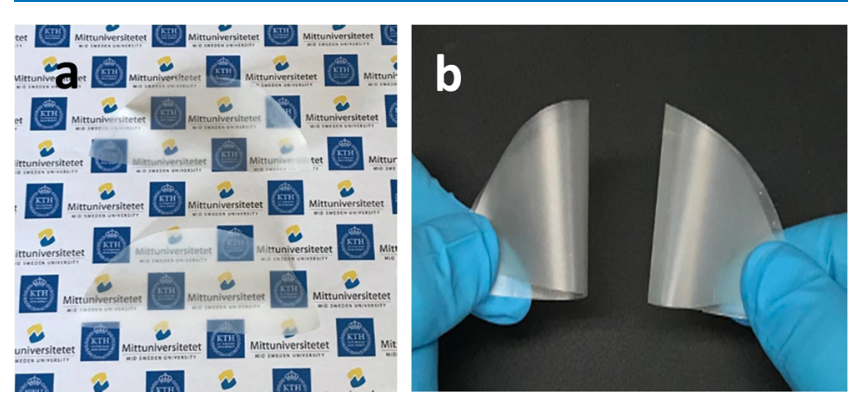

Figure 3. (a) MTM/CNF film top and TPSi-MTM/CNF film bottom. (b) MTM/CNF film left and TPSi-MTM/CNF film right.

Structure and Characterization of the MTM/CNF Nanocomposites. The successful modification was confirmed by contact angle measurements, IR analysis, and elemental analysis. In addition, elemental mapping of the MTM/CNF nanocomposites reveals a homogeneous distribution of carbon (CNF) and silicon (MTM) (Figure 4a,b) used as references. It also shows the fluorine (Figure $4 \mathrm{c}$ ) and sulfur (Figure 4d) distribution with the highest concentration being on the surface of the corresponding $(1 \mathrm{H}, 1 \mathrm{H}, 2 \mathrm{H}, 2 \mathrm{H}$ heptadecaflurodecyl)Si-MTM/CNF and TPSi-MTM/CNF films, respectively. This also supported by elemental analysis where the sulfur content of the TPSi-modified film is $0.61 \mathrm{wt} \%$ $(0.019 \mathrm{mmol} \mathrm{S})$, which is a ratio of 0.042 to the amount of glucose units $(0.45 \mathrm{mmol})$ present in the sample. Thus, only the outer surface is modified by the organofluorosilane agent. The cross-sections of the fractured surfaces of modified MTM/ $\mathrm{CNF}$ and unmodified MTM/CNF nanocomposite films obtained by uniaxial tension at $50 \% \mathrm{RH}$ were observed by field-emission scanning electron microscopy (FESEM) and micrographs are shown in Figure 4e. Both modified C16SiMTM/CNF and unmodified MTM/CNF nanocomposites show a lamellar microstructure. Previous XRD measurments of the MTM/CNF has shown that it resembles the brick-andmortar structure of nacre. ${ }^{17}$ The mechanical performance of the nanocomposites was investigated at $\mathrm{RH} 50 \%$ (Table 3). The tensile strength of the C16Si-MTM/CNF is $247 \pm 13$
Table 3. Mechanical Data of Various Films at 50\% RH

\begin{tabular}{clccc} 
entry & \multicolumn{1}{c}{ sample } & $\begin{array}{c}\text { E-modulus } \\
(\mathrm{GPa})\end{array}$ & $\begin{array}{c}\text { tensile strength } \\
(\mathrm{MPa})\end{array}$ & $\begin{array}{c}\text { strain at } \\
\text { break (\%) }\end{array}$ \\
1 & CNF film & $17.2 \pm 0.9$ & $277 \pm 15$ & $5.9 \pm 0.6$ \\
2 & clay-film & -- & - & -- \\
3 & MTM/CNF film & $24.8 \pm 2.5$ & $267 \pm 18$ & $2.7 \pm 0.7$ \\
4 & C16Si-MTM/ & $25.2 \pm 1.2$ & $247 \pm 13$ & $2.1 \pm 0.3$ \\
& CNF film & & & \\
\hline
\end{tabular}

$\mathrm{MPa}$ (E-modulus $=25.2 \pm 1.2 \mathrm{GPa}$, strain at break $=2.1 \pm$ $0.3 \%$, entry 4) and the strength of the MTM/CNF is $267 \pm 18$ $\mathrm{MPa}$ (E-modulus $=24.8 \pm 2.5 \mathrm{GPa}$, strain at break $=2.7 \pm$ $0.7 \%$, entry 3 ). Thus, the high strength of MTM/CNF films is maintained after the catalytic surface modifications. In comparison, the tensile strength of the pure CNF film is 277 $\pm 15 \mathrm{MPa}$ (E-modulus $=17.2 \pm 0.9 \mathrm{GPa}$, strain at break $=5.9$ $\pm 0.6 \%$, entry 1$)$ and for pure MTM is too brittle to measure (entry 2). This result is important and justifies the strategy to modify after the formation of the MTM/CNF nanocomposite since the approach of using hydrophobic CNF-nanoparticles to create films in water failed due to dispersion difficulties. It is the MTM/CNF core that contributes to the mechanical properties of the films and the surface modification is only a small percentage of the functional groups on the film surface. Moreover, at higher humidity (e.g., 90\% RH) the mechanical properties are significantly worse at the MTM/CNF core. ${ }^{33}$

The interaction between MTM and CNFs was further investigated by FTIR spectroscopy (Figure 5, see also the Supporting Information). We observed a similar frequency of vibrational bands for MTM/CNF (Figure 5a) and TPSiMTM/CNF (Figure 5b), dominated by strong hydrogen bonding only in cellulose at $3326 \mathrm{~cm}^{-1}$, whereas the MTM/ CNF interface is not expected to be identifiable in the spectrum. Under dry or even $50 \% \mathrm{RH}$ conditions, we still expect favorable MTM/CNF interface bonding from previous studies. The use of hydrophobic, surface-modified nanoparticles during processing and solidification would penalize the interface interactions between CNF and MTM. ${ }^{39}$ The IR also confirmed that (3-thiapropyl)thrimethoxy silane had been attached to the nanocomposite film at $2564 \mathrm{~cm}^{-1}$ (Figure $5 \mathrm{~b}$ ).

In order to investigate the surface, we performed atomic force microscopy (AFM) of the MTM/CNF and TPSi-MTM/ CNF nanocomposites, respectively (Figure 6). The AFM revealed that the roughness of the nanocomposite film increased from the $\mathrm{nm}$ scale to the $\mu \mathrm{m}$ scale after L-tartaric acid-catalyzed thiapropylsilane modification, which is proposed to be due to the catalytic silylation. However, when modifying a MTM/CNF nanocomposite film with a 2.2 times smoother
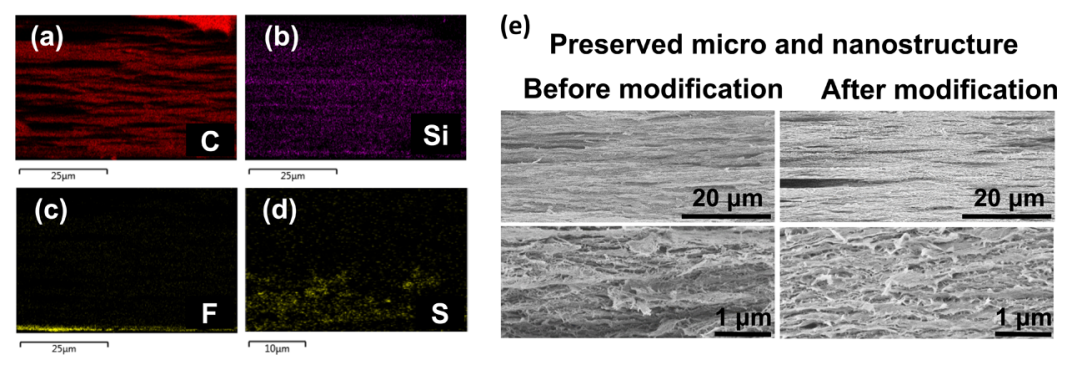

Figure 4. Elemental mapping $(\mathrm{a}-\mathrm{d}),(\mathrm{a})$ homogeneous distribution of carbon (CNF), (b) silicon (MTM), and (c) fluorine at the surface of $(1 \mathrm{H}, 1 \mathrm{H}, 2 \mathrm{H}, 2 \mathrm{H}$-heptadecaflurodecyl)Si-MTM/CNF. (d) Sulfur at the surface of TPSi-MTM/CNF. (e) Resulting cross-section of the MTM/CNF nanopaper before and after modification with $\mathrm{C} 16 \mathrm{Si}$. 


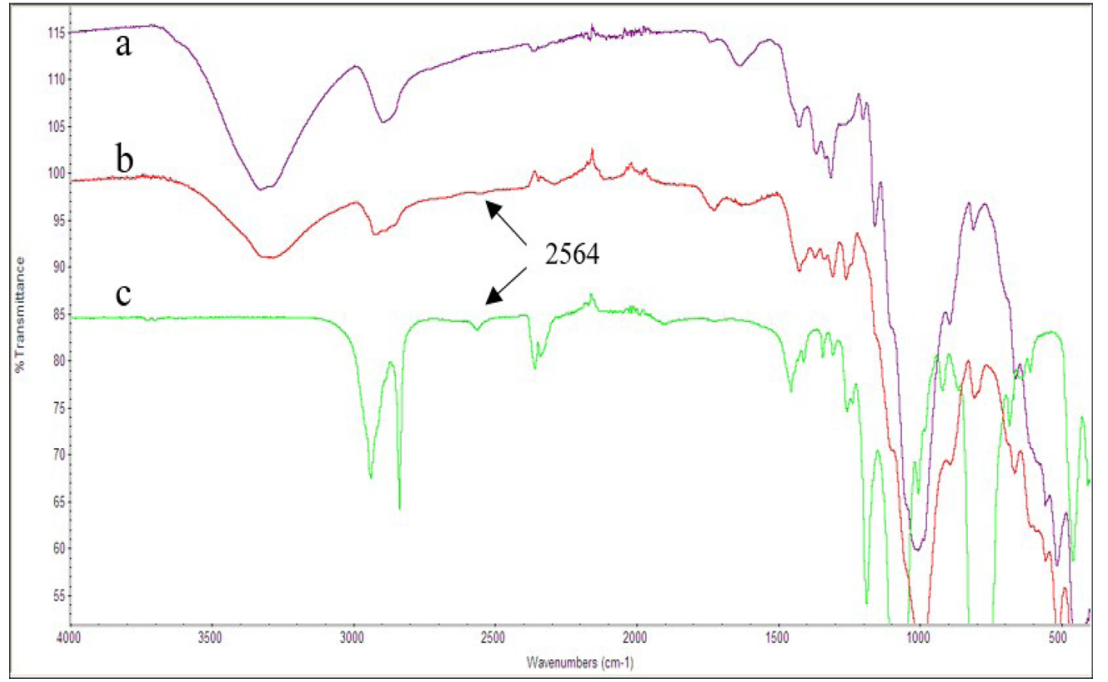

Figure 5. FTIR spectrum of the CNF/Clay nanocomposite. (a) MTM/CNF. (b) TPSi-MTM/CNF (c) (3-thiapropyl)trimethoxysilane (TPSi).

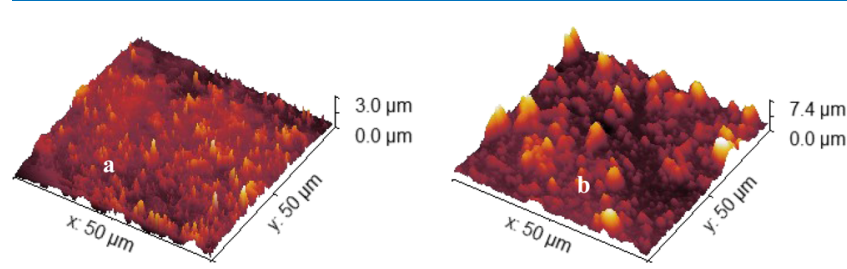

Figure 6. AFM images of nanocomposites. (a): MTM/CNF nanocomposite, RMS roughness (sq): $296 \mathrm{~nm}$, surface area: 3187 $\mu \mathrm{m}^{2}$ (b) TPSi-MTM/CNF, RMS roughness (sq): $1.028 \mu \mathrm{m}$, surface area: $4039 \mu \mathrm{m}^{2}$.

surface as compared to the one depicted in Figure 6a, a less rough TPSi-MTM/CNF surface was obtained as compared to the one shown in Figure $6 \mathrm{~b}$ and a lower contact angle was hence observed (see Figure S8). This is in accordance with the theory of the importance of surface roughness for the design of hydrophobic surfaces with a high contact angle.

\section{CONCLUSIONS}

In summary, we disclose a general organocatalytic strategy for the versatile functionalization and hydrophobization of nacremimetic nanocomposites. The merging of metal-free catalysis and the design of nacre-mimetic nanocomposite materials allows for facile and mild surface engineering of this important class of nanocomposites. The concept is demonstrated by the sustainable assembly of strong, transparent, hydrophobic, renewable, and flexible MTM/CNF films using several silanes with useful functionalities (e.g., $-\mathrm{SH},-\mathrm{NH}_{2}$, olefin, and aryl). In order to obtain transparent films, the organocatalytic silylation reactions were made after the assembly of the MTM/ CNF nanocomposites. Since there are a large variety of available silanes, it is possible to attach a plethora of molecules to nacre-mimetic nanocomposites using the disclosed strategy. Furthermore, the $-\mathrm{SH}$ and olefinic groups are excellent reactive groups for attachment of biopolymers and bioactive molecules. Such multifunctional combinations make these nanomaterials interesting for broader applications, such as drug delivery and tissue engineering, as well as packaging, construction, and advanced functional materials. Thus, further development of multifunctional nanocomposites inspired by the architecture of nacre is envisioned based on the disclosed strategy.

\section{EXPERIMENTAL SECTION}

General. Chemicals (e.g., hexadecyltrimethoxysilane (C16Si) from Sigma-Aldrich, (3-thiapropyl)trimethoxysilane (TPSi) from Sigma-Aldrich, $(1 \mathrm{H}, 1 \mathrm{H}, 2 \mathrm{H}, 2 \mathrm{H}-$ heptadecaflurodecyl)trimethoxysilane $(1 \mathrm{H}, 1 \mathrm{H}, 2 \mathrm{H}, 2 \mathrm{H}$-heptadecaflurodecyl)Si from TCI) and solvents were used as purchased without any further purification. Tartaric acid from Sigma-Aldrich and citric acid from Merck were dried in a desiccator over phosphorus pentoxide. Fourier-transformed infrared spectroscopy was recorded by Thermo Scientific NICOLET 6700 FTIR, Smart orbit, Diamond 30,000-200 $\mathrm{cm}^{-1}$ using transmission mode (160 scans) directly on the films. The AFM experiments were carried out with dimension icon and tapping mode with Bruker TAP525A as a cantilever. Resonance frequency for these experiments was $445 \mathrm{kHz}$ with a 0.32 lines/s scanning rate.

Materials. CNF. The CNF suspension (1.5 wt \%) in Milli$\mathrm{Q}$ water was prepared by high-pressure homogenization of an enzymatically pretreated sulfite pulp (Nordic AB, Sweden), as reported by Henriksson et al. ${ }^{23}$ Thus, pulp fibers were washed and beaten prior to addition of an endoglucanase under slight heating. After enzyme hydrolysis, the CNFs were washed and homogenized resulting in the 1.5 wt \% suspension.

Clay Suspension. A stable MTM suspension (approx. 0.35 wt $\%, \mathrm{Na}+$ Cloisite, BYK additives, Germany) was prepared by intense mixing and ultrasonication of MTM powder in Milli-Q water using a probe shear-mixer (Ultra-Turrax) for $30 \mathrm{~min}$. Notably, only the supernatant fraction of the MTM suspension after centrifugation (4500 rpm, $30 \mathrm{~min}$ ) was used and the aggregates were removed. Sonication/centrifugation cycles were repeated until all aggregates were removed, which is typically 3-4 times. The yield of the processes is $70 \%$.

Preparation of Nanocomposites. The MTM/CNF nanocomposites were prepared by filtration and drying, as reported Medina et al. ${ }^{17}$ Briefly, CNF water suspension was diluted to $0.15 \mathrm{wt} \%$ and sheared with an Ultra-Turrax apparatus. After $10 \mathrm{~min}$, the above MTM suspension was added and the mixture was mixed for 5 more min. The welldispersed aqueous MTM/CNF suspension was filtered with a microfiltration membrane with a pore size of $0.65 \mu \mathrm{m}$ for several hours. The resulting wet cake was dried in a hand sheet dryer (Rapid Kothen, Austria) under reduced pressure at 93 
${ }^{\circ} \mathrm{C}$ for $15 \mathrm{~min}$. The nanocomposite contained $20 \mathrm{wt} \% \mathrm{MTM}$ and 80 wt \% CNF.

Typical Procedure for Catalytic Surface Modification of Nanocomposites. The silane derivative (3 equivalents, based on the CNF) and nanocomposite (338 mg), which contains CNF $(270 \mathrm{mg}, 1.66 \mathrm{mmol}$ (based on glucose anhydrous unit), 1 equiv) were placed in an oven-dried reaction vessel containing L-tartaric acid ( $36 \mathrm{mg}, 0.24 \mathrm{mmol}, 5$ mol \%) and dry toluene $(50 \mathrm{~mL})$. After performing the reaction for $48 \mathrm{~h}$ at $95{ }^{\circ} \mathrm{C}$, the temperature was decreased to room temperature and the toluene was decanted. Next, the modified nanocomposite was washed with acetone $(4 \times 50$ $\mathrm{mL}$ ) and dried under reduced pressure.

Typical Procedure for Catalytic Surface Modification of CNF. A $31 \mathrm{~g}$ of suspension of CNF (1.6 wt \%, $500 \mathrm{mg}$ dry mass, $3 \mathrm{mmol}$ ) was homogenized in $\mathrm{EtOH}(95 \%, 60 \mathrm{~mL})$ for 1 $\mathrm{h}$ at $6000 \mathrm{rpm}$. The solvent was exchanged with EtOH (95\%, 3 $\times 60 \mathrm{~mL}$ ) using centrifugation. The CNF was next transferred to the reaction vessel in $\mathrm{EtOH}(95 \%, 30 \mathrm{~mL})$. Next, (3mercaptopropyl)trimethoxysilane $(1.4 \mathrm{~mL}, 7.5 \mathrm{mmol}, 2.5$ mequiv) and tartaric acid (23 $\mathrm{mg}, 0.15 \mathrm{mmol}, 5 \mathrm{~mol} \%$ ) were sequentially added. After refluxing the resulting reaction mixture for $24 \mathrm{~h}$, it was cooled down to room temperature and the modified CNF was washed with EtOH $(95 \%, 3 \times 60 \mathrm{~mL})$ using centrifugation.

Typical Procedure for Catalytic Surface Modification of MTM. Allyltrimethoxysilane $(0.34 \mathrm{~mL}, 2 \mathrm{mmol})$ and MTM $(500 \mathrm{mg})$ were placed in an oven-dried reaction vessel containing tartaric acid $(25 \mathrm{mg}, 5 \mathrm{wt} \%)$ and dry toluene (10 $\mathrm{mL}$ ). After stirring the reaction mixture at $80{ }^{\circ} \mathrm{C}$ for $6 \mathrm{~h}$, the temperature was decreased to room temperature and toluene was decanted. Next, the modified MTM was Soxhlet extracted (acetone, $24 \mathrm{~h}$ ) and dried under reduced pressure.

Contact Angle Measurements. The contact angle (CA) was recorded on a DAT 1100-FIBRO-system ab-SWEDEN and the measurement method is TAPPI T $558 \mathrm{pm}-95$. The instrument was in dynamic mode and the contact angles are given after $5 \mathrm{~min}$.

Field-Emission Scanning Electron Microscopy (FESEM). High-resolution micrographs of the sample surface and cross-sections were recorded using a Hitachi S4800 fieldemission scanning electron microscope (Japan). The typical accelerating voltage was $1 \mathrm{kV}$ and the working distance was 3$4 \mathrm{~mm}$. Additionally, elemental mapping was carried out using an energy-dispersive X-ray detector (Oxford Instruments, UK) at $15 \mathrm{kV}$ and high probe current.

Tensile Testing. Tensile properties of the modified and unmodified films were determined on a Universal Testing Machine, Instron 5944 (USA). The samples were first conditioned at $50 \%$ relative humidity and $22 \pm 1{ }^{\circ} \mathrm{C}$ for at least $40 \mathrm{~h}$. Rectangular strips of $3-5 \mathrm{~mm}$ in width were then cut and tested, using a gauge length of $22 \mathrm{~mm}$ and a strain rate of $0.1 \mathrm{~min}^{-1}$. The instrument was equipped with a noncontact video extensometer for strain measurement, and the load was measured with a $500 \mathrm{~N}$ load cell. All reported values are averaged data from at least five specimens.

Atomic Force Microscopy. The AFM experiments were carried out with dimension icon and tapping mode with Bruker TAP525A as a cantilever. Resonance frequency for these experiments was $445 \mathrm{kHz}$ with a 0.32 lines/s scanning rate.

\section{ASSOCIATED CONTENT}

\section{Supporting Information}

The Supporting Information is available free of charge at https://pubs.acs.org/doi/10.1021/acsomega.0c00978.

Detailed experimental procedures for fabrication of CNFs, fabrication of MTM suspension, preparation of MTM/CNF nanocomposites, procedures for organocatalytic surface modifications, IR spectra, and tensile stress measurements (PDF)

\section{AUTHOR INFORMATION}

\section{Corresponding Author}

Armando Córdova - Department of Natural Sciences, Mid Sweden University, SE-851 70 Sundsvall, Sweden; (i) orcid.org/0000-0001-9620-8698;

Email: armando.cordova@miun.se

\section{Authors}

Rana Alimohammadzadeh - Department of Natural Sciences, Mid Sweden University, SE-851 70 Sundsvall, Sweden

Lilian Medina - Department of Fiber and Polymer Technology, Wallenberg Wood Science Center, KTH Royal Institute of Technology, 10044 Stockholm, Sweden; O orcid.org/00000001-8547-9046

Luca Deiana - Department of Natural Sciences, Mid Sweden University, SE-851 70 Sundsvall, Sweden

Lars A. Berglund - Department of Fiber and Polymer Technology, Wallenberg Wood Science Center, KTH Royal Institute of Technology, 10044 Stockholm, Sweden; () orcid.org/0000-0001-5818-2378

Complete contact information is available at:

https://pubs.acs.org/10.1021/acsomega.0c00978

\section{Author Contributions}

R.A. and L.D. performed the catalytic functionalization, IR analyses, and contact angle measurements. R.A. and L.M. synthesized the MTM/CNF nanocomposite films. L.M. synthesized the CNF, performed the elemental mapping experiments, FESEM, and tensile strength measurements. The AFM measurements were made at KTH assisted by R.A. The manuscript was written through contributions by all the authors. The research was coordinated and led by L.B. and A.C. who also edited the manuscript. All the authors proofread the manuscript and have given approval to the final version of the manuscript.

\section{Notes}

The authors declare no competing financial interest.

\section{ACKNOWLEDGMENTS}

R.A., L.D., and A.C. acknowledge funding from the Swedish Research Council (VR), the European Union, and the Knowledge Foundation. L.M. and L.A.B. acknowledge funding from the Knut and Alice Wallenberg Foundation through the Wallenberg Wood Science Center and the Swedish Foundation for Strategic Research (SSF) through the FiReFoam project (RMA11-0065). This research has been supported by Treesearch.se.

\section{REFERENCES}

(1) Mayer, G. Rigid Biological Systems as Models for Synthetic Composites. Science 2005, 310, 1144. 
(2) Studart, A. R. Towards High-Performance Bioinspired Composites. Adv. Mater. 2012, 24, 5024.

(3) Liu, K.; Jiang, L. Bio-Inspired Design of Multiscale Structures for Function Integration. Nano Today 2011, 6, 155.

(4) Kamat, S.; Su, X.; Ballarini, R.; Heuer, A. H. Structural Basis for the Fracture Toughness of the Shell of the Conch Strombus Gigas. Nature 2000, 405, 1036.

(5) Cartwright, J. H. E.; Checa, A. G. The Dynamics of Nacre SelfAssembly. J. R. Soc., Interface 2006, 4, 491.

(6) Li, X.; Chang, W.-C.; Chao, Y. J.; Wang, R.; Chang, M. Nanoscale Structural and Mechanical Characterization of a Natural Nanocomposite Material: The Shell of Red Abalone. Nano Lett. 2004, 4, 613 .

(7) Tang, Z.; Kotov, N. A.; Magonov, S.; Ozturk, B. Nanostructured Artificial Nacre. Nat. Mater. 2003, 2, 413.

(8) Podsiadlo, P.; Kaushik, A. K.; Arruda, E. M.; Waas, A. M.; Shim, B. S.; Xu, J.; Nandivada, H.; Pumplin, B. G.; Lahann, J.; Ramamoorthy, A.; Kotov, N. A. Ultrastrong and Stiff Layered polymer Nanocomposites. Science 2007, 318, 80.

(9) Liang, B.; Zhao, H.; Zhang, Q.; Fan, Y.; Yue, Y.; Yin, P.; Guo, L. $\mathrm{Ca}^{2+}$ Enhanced Nacre-Inspired Montmorillonite-Alginate Film with Superior Mechanical, Transparent, Fire Retardancy, and Shape Memory Properties. ACS Appl. Mater. Interfaces 2016, 8, 28816.

(10) Sehaqui, H.; Kochumalayil, J.; Liu, A.; Zimmermann, T.; Berglund, L. A. Multifunctional Nanoclay Hybrids of High Toughness, Thermal and Barrier Performances. ACS Appl. Mater. Interfaces 2013, 5, 7613.

(11) Bonderer, L. J.; Studart, A. R.; Gauckler, L. J. Bioinspired Design and Assembly of Platelet Reinforced Polymer Films. Science 2008, 319, 1069.

(12) Gao, F. Clay/Polymer Composites: The Story. Mater. Today 2004, 50.

(13) Nanoclay Reinforced Polymer Composites: Nanocomposites and Biocomposites. In Engineering Materials; Jawaid, M.; el Kacem Qaiss, A.; Bouhfid, R. Eds.; Springer Nature, Singapore 2016.

(14) Walther, A.; Bjurhager, I.; Malho, J.-M.; Ruokolainen, J.; Berglund, L.; Ikkala, O. Supramolecular Control of Stiffness and Strength in Lightweight High-Performance Nacre-Mimetic Paper with Fire-Shielding Properties. Angew. Chem., Int. Ed. 2010, 49, 6448.

(15) Das, P.; Malho, J.-M.; Rahimi, K.; Schacher, F. H.; Wang, B.; Demco, D. E.; Walther, A. Nacre-Mimetics with Synthetic Nanoclays up to Ultrahigh Aspect Ratios. Nat. Commun 2015, 6, 5967.

(16) Sehaqui, H.; Liu, A.; Zhou, Q.; Berglund, L. A. Fast Preparation

Procedure for Large Flat Cellulose and Cellulose/Inorganic NanoPaper Structures. Biomacromolecules 2010, 11, 2195.

(17) Medina, L.; Nishiyama, Y.; Daicho, K.; Saito, T.; Yan, M.; Berglund, L. A. Nanostructure and Properties of Nacre-Inspired Clay/ Cellulose Nanocomposites-Synchrotron X-ray Scattering Analysis. Macromolecules 2019, 52, 3131.

(18) Wu, C.-N.; Saito, T.; Fujisawa, S.; Fukuzumi, H.; Isogai, A. Ultrastrong and High Gas-Barrier Nanocellulose/Clay-Layered Composites. Biomacromolecules 2012, 13, 1927.

(19) Aulin, C.; Salazar-Alvarez, G.; Lindström, T. High Strength, Flexible and Transparent Nanofibrillated Cellulose-Nanoclay Biohybrid Films with Tunable Oxygen and Water Vapor Permeability. Nanoscale 2012, 4, 6622.

(20) Yao, K.; Huang, S.; Tang, H.; Xu, Y.; Buntkowsky, G.; Berglund, L. A.; Zhou, Q. Bioinspired Interface Engineering for Moisture Resistance in Nacre-Mimetic Cellulose Nanofibrils/Clay Nanocomposites. ACS Appl. Mater. Interfaces 2016, 9, 20169.

(21) Liu, A.; Berglund, L. A. Clay Nanopaper Composites of NacreLike Structure Based on montmorrilonite and cellulose nanofibersImprovements due to Chitosan Addition. Carbohydr. Polym. 2012, 87, 53.

(22) Wang, J.; Cheng, Q.; Lin, L.; Jiang, L. Synergistic Toughening of Bioinspired Poly(vinyl alcohol)-Clay-Nanofibrillar Cellulose Artificial Nacre. ACS Nano 2014, 8, 2739.

(23) Henriksson, M.; Henriksson, G.; Berglund, L. A.; Lindström, T. An Environmentally Friendly Method For Enzyme-Assisted Prepara- tion of Microfibrillated Cellulose (MFC) Nanofibers. Eur. Polym. J. 2007, 43, 3434.

(24) Saito, T.; Nishiyama, Y.; Putaux, J.-L.; Vignon, M.; Isogai, A. Homogeneous Suspensions of Individualized Microfibrils from TEMPO-Catalyzed Oxidation of Native Cellulose. Biomacromolecules 2006, 7, 1687.

(25) Nakagaito, A. N.; Yano, H. The effect of morphological changes from pulp fiber towards nano-scale fibrillated cellulose on the mechanical properties of high-strength plant fiber based composites. Appl. Phys. A 2004, 78, 547.

(26) Sakurada, I.; Nukushina, Y.; Ito, T. Experimental Determination of the Elastic Modulus of Crystalline Regions in Oriented Polymers. J. Polym. Sci. 1962, 57, 651.

(27) Saito, T.; Kuramae, R.; Wohlert, J.; Berglund, L. A.; Isogai, A. An Ultrastrong Nanofibrillar Biomaterial: The Strength of Single Cellulose Nanofibrils Revealed via Sonication-Induced Fragmentation. Biomacromolecules 2012, 14, 248-253.

(28) Rol, F.; Belgacem, M. N.; Gandini, A.; Bras, J. Recent Advances in Surface-Modified Cellulose Nanofibrils. Prog. Polym. Sci. 2019, 88, 241.

(29) Habibi, Y. Key Advances in the Chemical Modification of Nanocelluloses. Chem. Soc. Rev. 2014, 43, 1519.

(30) Du, G.; Mao, A.; Yu, J.; Hou, J.; Zhao, N.; Han, J.; Zhao, Q.; Gao, W.; Xie, T.; Bai, H. Nacre-Mimetic Composite with Intrinsic Self-Healing and Shape-Programming Capability. Nat. Commun. 2019, 10, 800.

(31) Zhao, N.; Yang, M.; Zhao, Q.; Gao, W.; Xie, T.; Bai, H. Superstretchable Nacre-Mimetic Graphene/Poly(vinyl alcohol) Composite Film Based on Interfacial Architectural Engineering. ACS Nano 2017, 11, 4777.

(32) Zhao, H.; Yang, Z.; Guo, L. Nacre-Inspired Composites with Different Macroscopic Dimensions: Strategies for Improved Mechanical Performance and Applications. NPG Asia Mater. 2018, 10, 1.

(33) Medina, L.; Ansari, F.; Carosio, F.; Salajkova, M.; Berglund, L. A. Nanocomposites from Clay, Cellulose Nanofibrils, and Epoxy with Improved Moisture Stability for Coatings and Semistructural Applications. ACS Appl. Nano Mater. 2019, 2, 3117.

(34) Wu, Q.; Guo, D.; Zhang, Y.; Zhao, H.; Chen, D.; Nai, J.; Liang, J.; Li, X.; Sun, N.; Guo, L. Facile and Universal Superhydrophobic Modification to Fabricate Waterborne, Multifunctional NacreMimetic Films With Excellent Stability. ACS Appl. Mater. Interfaces 2014, 6, 20597.

(35) Xu, L.-P.; Peng, J.; Liu, Y.; Wen, Y.; Zhang, X.; Jiang, L.; Wang, S. Nacre-Inspired Design of Mechanical Stable Coating with Underwater Superoleophobicity. ACS Nano 2013, 7, 5077.

(36) Kochumalayil, J. J.; Morimune, S.; Nishino, T.; Ikkala, O.; Walther, A.; Berglund, L. A. Nacre-Mimetic Clay/Xyloglucan Bionanocomposites: A Chemical Modification Route for Hygromechanical Performance at High Humidity. Biomacromolecules 2013, $14,3842$.

(37) Das, P.; Walther, A. Ionic supramolecular bonds preserve mechanical properties and enable synergetic performance at high humidity in water-borne, self-assembled nacre-mimetics. Nanoscale 2013, 5, 9348.

(38) Hafrén, J.; Córdova, A. Direct Organocatalytic Polymerization from Cellulose Fibers. Macromol. Rapid Commun. 2005, 26, 82.

(39) Hafrén, J.; Córdova, A. Direct Bronsted acid-catalyzed derivatization of cellulose with poly(L-lactic acid) and D-mandelic acid. Nord. Pulp Paper Res. J. 2007, 22, 184.

(40) Córdova, A.; Hafren, J.; Samec, J.; Enquist, K. Sheet-Formed Fibrous Material with Improved Strength Property, PCT/EP2009/ 05396. WO 2009121926 A1, 2008.

(41) Hafrén, J.; Córdova, A. Organoclick: Applied Eco-Friendly and Metal-Free Catalysis for Wood and Fiber Modifications. In Industrial Biorenewables: A practical viewpoint", Domínguez de María, P., John Wiley \& Sons Inc.; Hoboken, NJ 2016.

(42) Afewerki, S.; Alimohammadzadeh, R.; Osong, S. H.; Tai, C.-W.; Engstrand, P.; Córdova, A. Sustainable Design for the Direct 
Fabrication and Highly Versatile Functionalization of Nanocelluloses. Global Challenges 2017, 1, 1700045.

(43) Córdova, A.; Afewerki, S.; Alimohammadzadeh, R.; Sanhueza, I.; Tai, C.-W.; Osong, S. H.; Engstrand, P.; Ibrahem, I. A sustainable strategy for production and functionalization of nanocelluloses. Pure Appl. Chem. 2019, 91, 865.

(44) It should be noted that we also separately modified the CNF with thiapropylsilane (TPSi) and MTM with allylsilane (AllylSi), respectively, using L-tartaric acid as the catalyst (see the Supporting Information). The catalytic reaction worked well and gave the corresponding hydrophobic TPSi-CNF and AllylSi-MTM, respectively. Thus, MTM can also be highly functionalized by organocatalysis, which should find application in a lot of different areas ranging from catalysis to toxic metal purification (For a review see: Varadwaj, G. B. B.; Parida, K.; Nyamori, V. O. Inorg. Chem. Front. 2016, 3, 1100.). However, trying to prepare a transparent AllylSiMTM/TPSi-CNF nanocomposite in organic solvent (e.g., EtOH) using the above described filtration technique failed. Instead agglomeration occurred and a white film with TPSi and AllylSi groups was formed.

(45) Das, P.; Thomas, H.; Moeller, M.; Walther, A. Large-scale, Thick, Self-assembled, Nacre-mimetic Brick-Walls as Fire Barrier Coatings on Textiles. Sci. Rep. 2017, 7, 39910.

(46) Kolb, H. C.; Finn, M. G.; Sharpless, K. B. Click Chemistry: Diverse Chemical Function from a Few Good Reactions. Angew. Chem., Int. Ed. 2001, 40, 2004.

(47) Hoyle, C. E.; Bowman, C. N. Thiol-Ene Click Chemistry. Angew. Chem., Int. Ed. 2010, 49, 1540.

(48) Zhao, G.-L.; Hafrén, J.; Deiana, L.; Córdova, A. Heterogeneous "Organoclick" Derivatization of Polysaccharides: Photochemical Thiol-ene Click Modification of Solid Cellulose. Macromol. Rapid Commun 2010, 31, 740.

(49) Huang, J. L.; Li, C. J.; Gray, D. G. Functionalization of cellulose nanocrystal films via "thiol-ene" click reaction. RSC Adv. 2014, 4, 6965. 